\title{
On-demand Connection Establishment Scheme to Increase the Capacity of BLE Networks
}

\author{
Yejin Shin and Soonuk Seol* \\ School of Electrical, Electronics and Communication Engineering \\ Korea University of Technology and Education (KOREATECH) \\ yepp1252@koreatech.ac.kr, suseol@koreatech.ac.kr
}

\begin{abstract}
Bluetooth was originally designed for continuous, streaming data applications that exchange a lot of data at a close range. With standardization of mesh networking capability, Bluetooth Low Energy (BLE) has attracted attention as a main protocol in the Internet of Things (IoT) applications, such as a smart home and a smart factory. However, in Bluetooth communication, a single device can only connect to a maximum of seven devices at the same time. This limitation also affects various IoT applications where many devices need to be connected to a single gateway within a one-hop range. In this paper, we propose a method to improve the device capacity in BLE-based networks. Our method establishes a connection between a BLE device and a gateway only when data exchange is required. For this, we utilize the Advertising Event message to recognize whether a device requires a connection. In addition, we design our method to make the connection between devices easier by using the contents of advertising packet. If our method is applied to an application that requires communication intermittently (or only when requested by user) such as monitoring or remote control, devices will be able to communicate with the gateway stably, even if there are numerous devices in the system.
\end{abstract}

Keywords: Bluetooth, Bluetooth Low Energy, BLE, Advertising Event, Beacon, iBeacon

\section{Introduction}

As smart home technology evolves, more and more home appliances are interconnected. The communication of a smart home is mainly performed indoors, and does not require wide coverage in most homes. Accordingly, most of the smart home products are connected to a single gateway (or a single hub) using a short range communication, and the gateway is connected to the Internet. Therefore, it is possible to provide remote monitoring and control functions or to control home appliances directly by using a hub through a smart home.

Many previous studies [1-5] have applied Bluetooth as a communication technology between devices and a gateway, to reduce the cost of the product and the power consumption of devices. However, in Bluetooth communication, a single device can only connect to a maximum of seven devices at the same time this limitation also affects various IoT applications. Therefore, it is difficult to communicate smoothly in a situation where many devices need to be connected to a single gateway. To overcome this problem, a large amount of research using advertising events of Bluetooth 4.0 [6-8] are being studied. Even if advertising events do not establish any connections between devices, because they broadcast control signals through advertising packets, so there is no limit to the number of devices that can be connected. However, broadcast link has problems that are vulnerable to security and it is not guaranteed to be reliable in transmission. In addition, advertising packets have difficulty in transmitting a lot of data at one time because the maximum payload is 31 bytes. There is also a limitation in bi-directional communication.

Received (September 8, 2017), Review Result (December 1, 2017), Accepted (December 7, 2017) 
In this paper, we propose a method to improve the device capacity in the BLE-based networks. To do this, we use advertising events to connect to the gateway only in situations requiring communication. In addition, we design a method to make the connection between devices easier by using the contents of an advertising packet. If our method is applied to an application that requires communication intermittently (or only when requested by user) such as monitoring or remote control, all devices will be able to communicate with the gateway stably even if there are numerous devices in the system. This improves device capacity in BLE-based networks while providing the reliability of inter-device communication.

The rest of this paper is structured as follows. The following section covers related works. Section 3 presents an on-demand connection establishment scheme to improve a device capacity in the BLE-based networks. Finally, Section 4 concludes this paper.

\section{Related Works}

\subsection{A Method for Configuration of Smart Home Network}

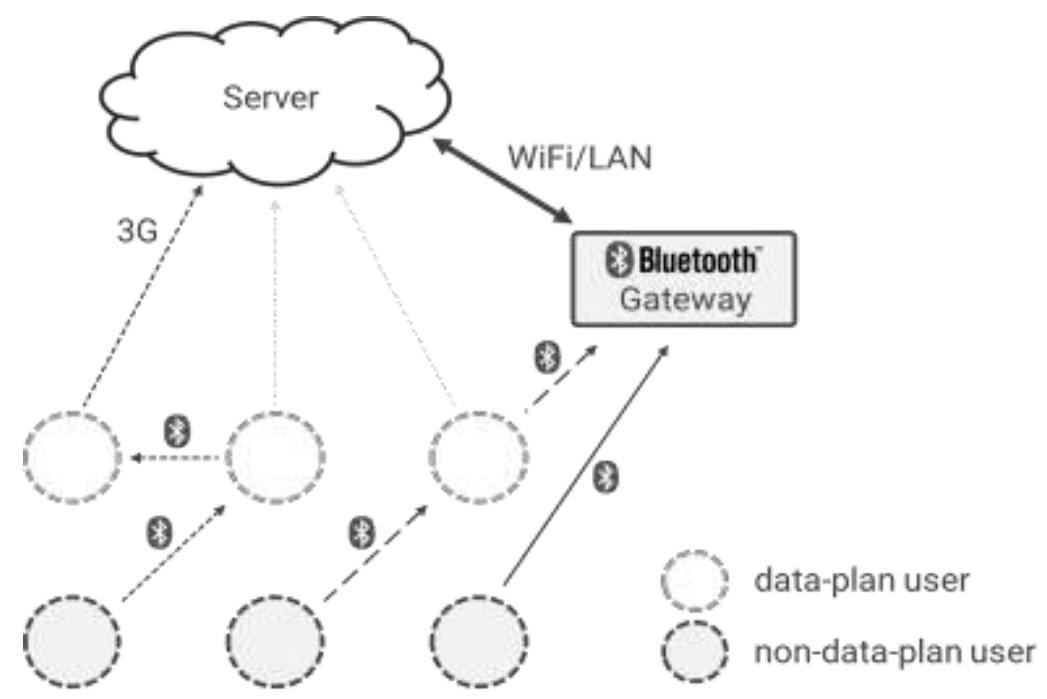

Figure 1. The Basic Idea of effSense [1]

Many previous studies [1-5] have applied Bluetooth as a communication technology between devices and a gateway, to reduce the cost of the product and the power consumption of devices. The authors of [1] proposes an effSense mechanism for uploading sensed data that has been collected from various devices via a Bluetooth gateway to the server. With a Bluetooth gateway, devices that can be directly connected to the Internet are able to save energy. In addition, devices that cannot be directly connected to the Internet are able to reduce communication costs. Figure 1 shows a simple example to illustrate the basic of effSense.

Recently, a lot of research using advertising events of Bluetooth 4.0 [6-8] is being studied. Sung-Woo Ahn's study [6] proposes a smart attendance confirmation system using advertising events based on Bluetooth Low Energy (BLE). The beacon devices periodically transmit advertisement packets, and students who receive the packets can check their attendance through the smartphone application. This scheme allows many devices to receive advertising packets at the same time. On the other hand, since devices cannot transmit, this scheme can be applied only to applications requiring unidirectional communication. 


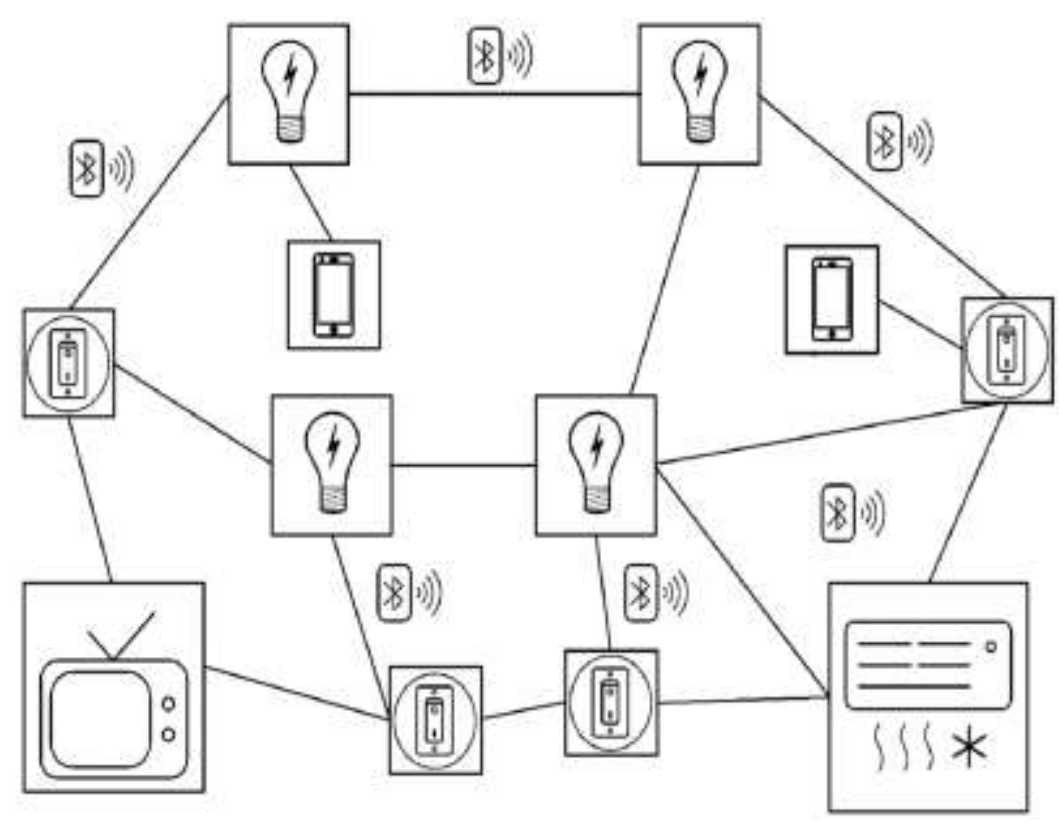

Figure 2. An Example of a Bluetooth Mesh Network [11]

With the standardization $[9,10]$ of mesh networking capability, BLE has attracted attention as a main protocol in the IoT applications, such as a smart home and a smart factory. An example of a Bluetooth mesh network can be seen in Figure 2. Uniqon Inc. [12] launched a product with longer communication range by sharing data among Bluetooth devices through BLE mesh networking. However, in Bluetooth communication, a single device can only connect to a maximum of seven devices at the same time. Therefore, it is difficult to communicate smoothly in a situation where many devices need to be connected to a single gateway within a one-hop range. In this paper, we propose a method to improve the device capacity in the BLE-based networks.

\subsection{Typical Operational Procedures of Bluetooth}

Figure 3 shows the typical operation procedure for creating a Bluetooth connection between two devices. As Bluetooth is an ad-hoc wireless communications technology, there are some operational procedures so that the subsequent communications can take place. A device may be engaged in a number of these procedures and modes:

2.2.1. Inquiry (Discovering): Bluetooth devices use the inquiry procedure to discover the other devices, or to be discovered by devices in their locality. A Bluetooth device that tries to find other nearby devices is known as an inquiring device. An inquiring device actively sends inquiry requests. Bluetooth devices that are available to be found are known as discoverable devices. Discoverable devices listen for inquiry requests and send responses. 


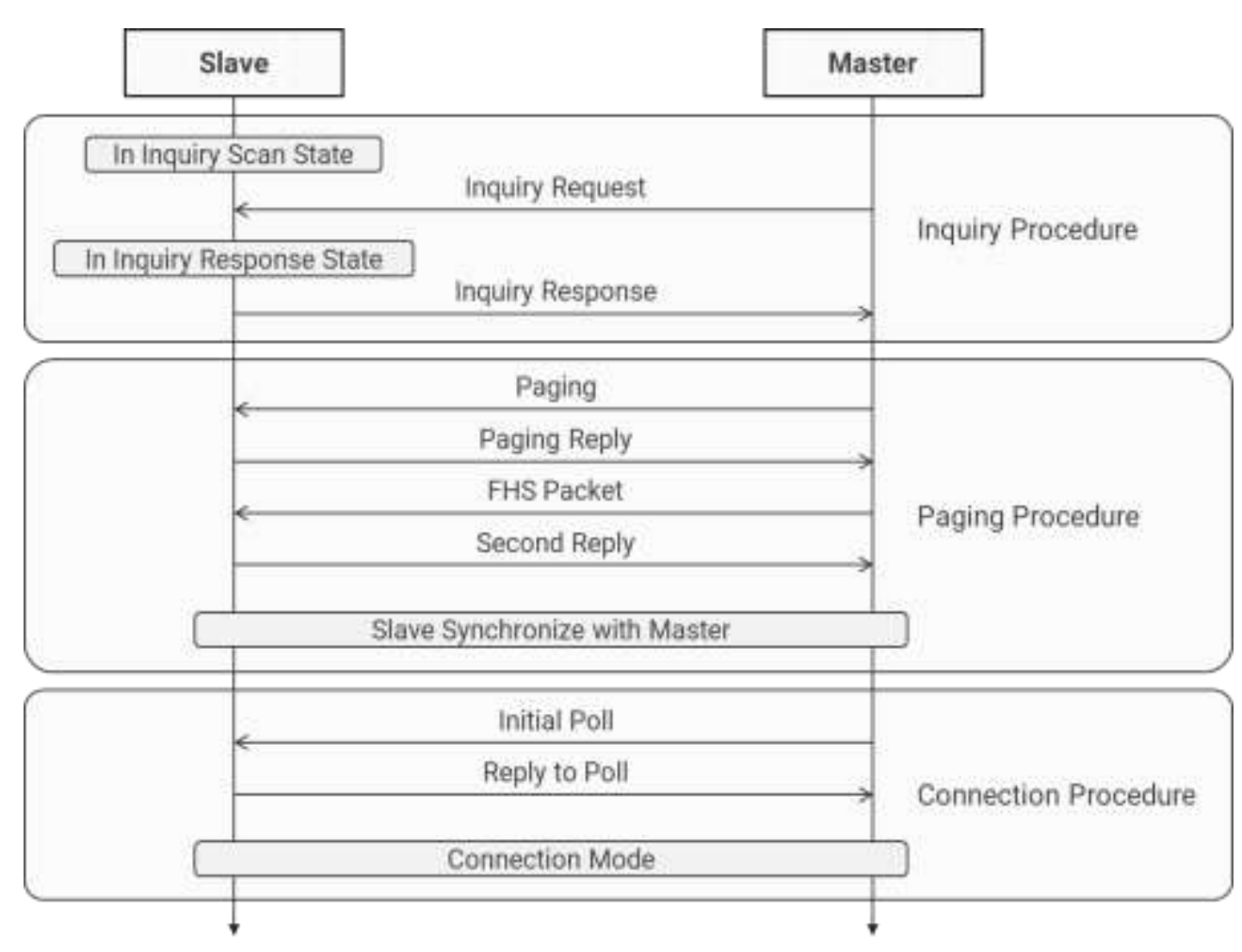

Figure 3. Connection Process of Bluetooth [13]

2.2.2. Paging (Connecting): The paging procedure for forming connections requires that one Bluetooth device carries out the page (connection) procedure while the other Bluetooth device is scanning the page. Because the procedure is targeted, the page procedure is only responded to by one specified Bluetooth device. Both paging and connectable device may already be connected to other Bluetooth devices.

2.2.3. Connection: There is a physical link between the devices after a successful paging procedure. While connected a device can change the modes of the physical and logical links (e.g., reduced power mode). It is also possible for the device to carry out inquiry, paging or scanning procedures or to be connected to other Bluetooth devices without needing to disconnect from the original physical channel [14].

\section{Design and Implementation of the System}

\subsection{Architecture of the System}

The proposed system can be used in applications in which many slaves are connected to a single gateway as shown in Figure 4. Our system improves the device capacity of the gateway by using advertising events to connect to the gateway only in situations requiring communication. In this system, slaves (end devices) operate as an advertiser that continuously broadcast advertising packets. And a master (the gateway) operates as a scanner to scan advertising packets. 


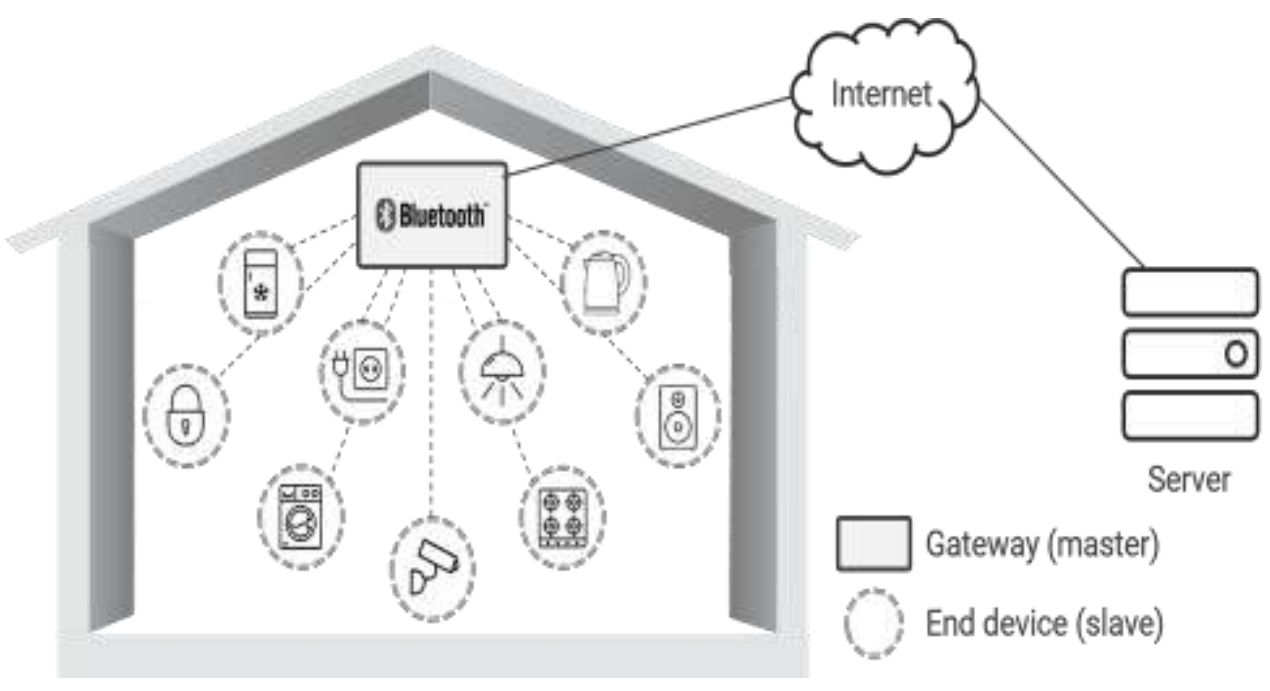

Figure 4. System Architecture

The gateway in the proposed system is connected to various home appliances (end devices) via Bluetooth. The home appliances can be controlled using the gateway. In addition, they can be remotely controlled by connecting to the Internet via the gateway. If a smart home system applies our on-demand connection establishment scheme, the gateway can accommodate all devices even if there is a large number of home appliances that we want to control. Our scheme is able to be applied not only to a smart home but also to various IoT applications such as a smart factory.

We designed and developed a platform that can easily connect legacy devices to the Internet via Bluetooth in our previous studies [15-17]. This allows legacy devices to be expanded to IoT devices at a low-cost without replacing existing devices. Details are addressed in Section 3.4.

\subsection{Message Composition}

Apple Inc. developed iBeacon [18] specification for transmitting data by using the "data" field of the BLE Advertising packet [14]. The iBeacon packet contains 16 bytes of "Proximity UUID" field, which is an identifier used to identify devices. In this paper, we design a message using "Proximity UUID" fields of iBeacon packet. Figure 5 shows the message format.

Table 1. Fields of the Advertising Packet

\begin{tabular}{|c|c|}
\hline Fields & Descriptions \\
\hline Product Type & $\begin{array}{c}\text { Identifies the slave which the master should connect by recognizing } \\
\text { the product in the same service }\end{array}$ \\
\hline State & \begin{tabular}{c} 
Indicates whether the slave requires a connection at that time or not \\
\hline MAC Address
\end{tabular} \\
\hline
\end{tabular}




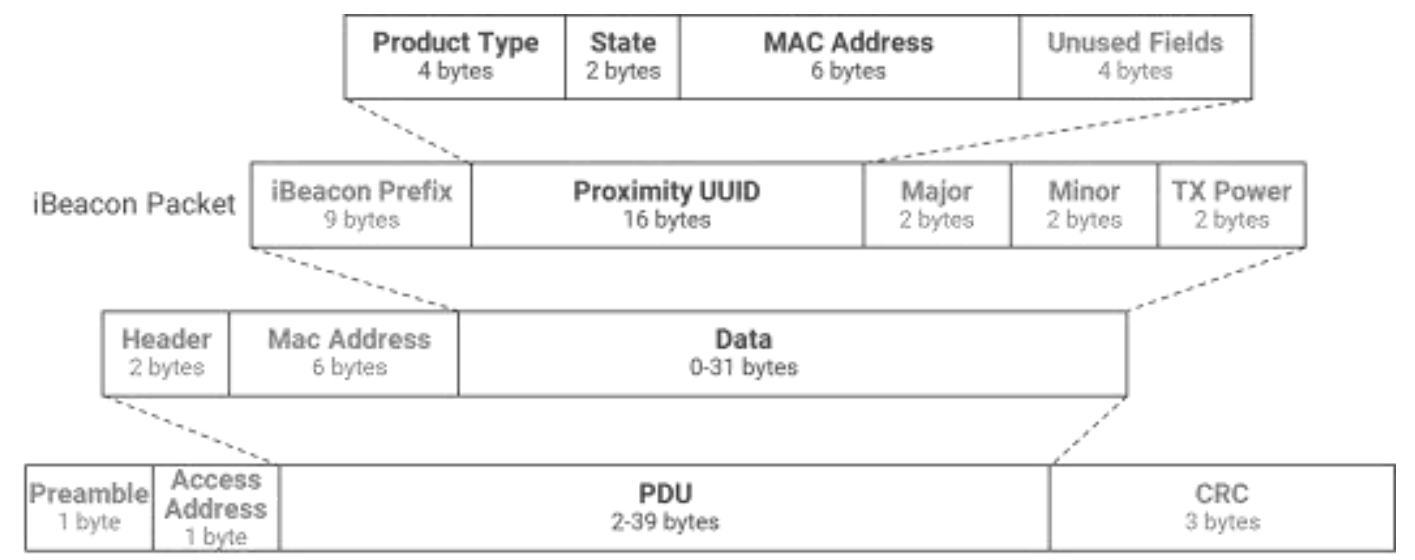

BLE Advertising Packet

Figure 5. Message Format

The iBeacon's UUID of the advertising packet broadcasted by the slave consists of the "product type", "state", and "MAC address" fields. Table 1 shows the description of each field. The "product type" is a unique character assigned to identify the slave which the master should connect among a large number of Bluetooth devices that may exist around. The "state" indicates whether the slave requires a connection at that time. The "MAC address" is a field for informing the slave's MAC address to the master, so that the master can connect to the slave directly.

The slave sets the "state" field of the advertising packet to 0 if there is no data to send. On the other hand, if there is data to send, the slave sets the "state" field to 1 and sets its MAC address in the "MAC address" field of the packet. The master verifies the contents of the scanned advertising packet if the "product type" is the same as its own. The master ignores it if the "state" field of the scanned packet is set to 0 . On the other hand, the master understands that the slave has data to send if the field of the packet is set to 1 . Therefore, the master can recognize the slave that needs a connection, and connect to that slave by using the MAC address.

Usually, many devices need a lot of steps to establish a Bluetooth connection; discovering the peripheral devices, checking the list of discovered devices, selecting the device to connect from the list, and so on. However, there is no way to check the list of discovered devices for devices that do not have a display. In this case, by using the proposed method, it is possible to accurately recognize the slave to be connected at this time by utilizing the state and the "product type" fields. Therefore, there is an advantage that devices without a display can also be connected to a desired device.

\subsection{Operational Procedures of the Proposed System}

3.3.1. Data Transmission Process from Slave to Master: Figure 6 shows the connection procedure when the slave has data to send to the master. The slave usually broadcasts the advertisement packet with the "state" field value set to 0 . If there is data to send, the slave changes the value of the "state" field to 1 . And then, the slave sets its own MAC address in the "MAC address" field. This process can also be performed when the slave is first registered with the system. 


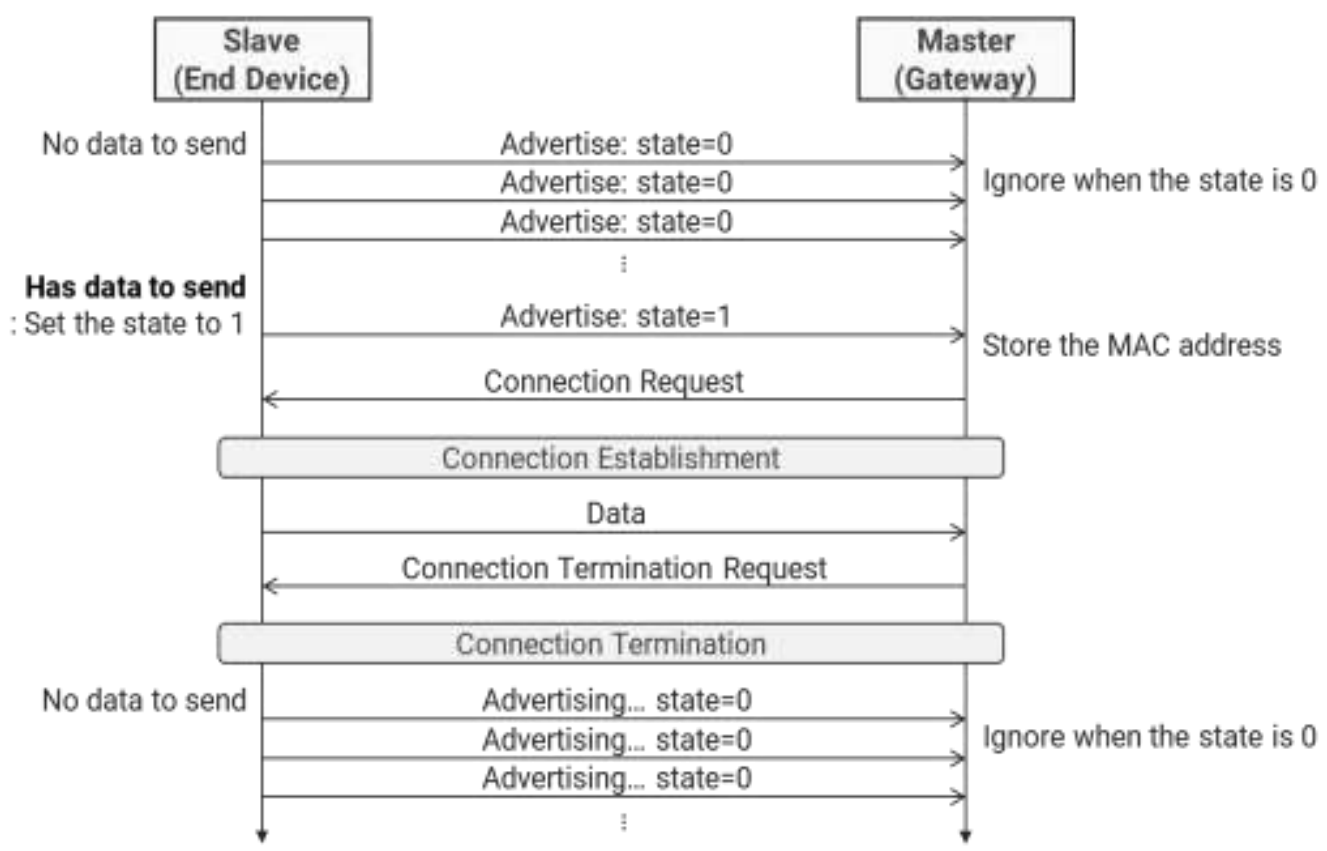

Figure 6. Data Transmission Process from Slave to Master

The master periodically scans the advertising packets. If the master scans a packet that's "state" field is at 1, the master recognizes that the slave has data to send and establishes a connection to the slave's MAC address. At this time, the master stores the slave's MAC address to be used in a situation where the master needs to send data to the slave at a later time.

Once the connection between the master and the slave has been established, the slave transfers the data to the master. After the data transfer is completed, the master terminates the connection with the slave. The slave again broadcasts the advertising packet by modifying the value of the "state" field to 0 .

3.3.2. Data Transmission Process from Master to Slave: Figure 7 shows the connection procedure when the master has data to send to the slave. If there is no data to send, the slave broadcasts the advertisement packet with the "state" field value set to 0 . The master ignores it if the "state" field of the scanned packet is set to 0 .

When the master has data to send to the slave, the master finds the destination MAC address from the stored address list. And then, the master establishes a connection using the MAC address that found. If the connection is established successfully, the master sends the data to the slave. When the data transfer is completed, the master terminates the connection with the slave. After the connection is terminated, the slave starts to broadcast the advertising packet again. 


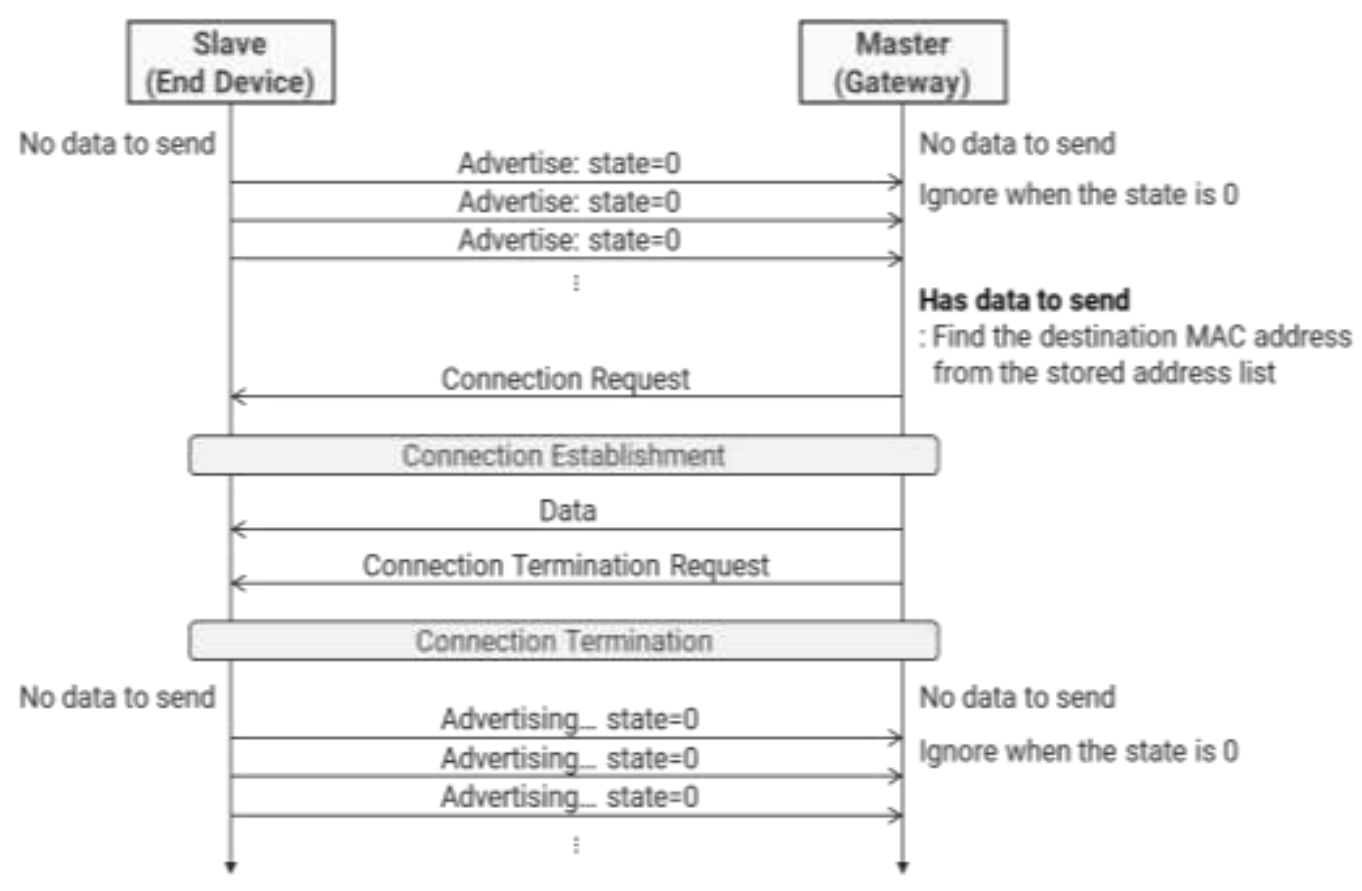

Figure 7. Data Transmission Process from Master to Slave

\section{Conclusions and Future Works}

In this paper, we proposed an on-demand connection establishment scheme to improve a device capacity in the BLE-based networks. To do this, we use advertising events to connect to the gateway only in situations requiring communication. In addition, we design a method to make the connection between devices easier by using the contents of an advertising packet. If our method is applied to an application that requires communication intermittently (or only when requested by a user) such as monitoring or remote control, all devices will be able to communicate with the gateway stably even if there are numerous devices in the system. This improves the device capacity in BLE-based smart home networks while providing the reliability and security of inter-device communication.

As future work, we intend to consider a method to enhance security of this system. In addition, we plan to evaluate the capacity of our system by applying the method to practical applications.

\section{Acknowledgement}

This work was supported by the National Research Foundation of Korea(NRF) grant funded by the Korea government(MSIP) (No. NRF-2016R1E1A2A02946448).

\section{References}

[1] L. Wang, D. Zhang and H. Xiong, "effSense : Energy-Efficient and Cost-Effective Data Uploading in Mobile Crowdsensing", Proceedings of the 2013 ACM conference on Pervasive and ubiquitous computing adjunct publication (UbiComp '13), (2013) September 8-12; Zurich, Switzerland.

[2] P. Angove, M. O'Grady, J. Hayes, B. O'Flynn, G. M. O'Hare and D. Diamond, “A Mobile Gateway for Remote Interaction with Wireless Sensor networks”, IEEE Sensors Journal, vol. 11, no. 12, (2011), pp.33093310.

[3] S. Raza, P. Misra, Z. He and T. Voigt, "Bluetooth Smart: An Enabling Technology for the Internet of Things", Wireless and Mobile Computing, Networking and Communications (WiMob 2015), 2015 IEEE 11th International Conference on, (2015) October 19-21; Abu Dhabi, UAE.

[4] T. H. Laine, C. Lee and H. Suk, "Mobile gateway for ubiquitous health care system using Zigbee and Bluetooth", The Eighth International Conference on Innovative Mobile and Internet Services in Ubiquitous Computing (IMIS-2014), (2014) July 2-4; Birmingham, UK. 
[5] S. I. Kang and A. S. Oh, "A Design and Implementation of Mobile Healthcare System based on Smart Gateway", Journal of the Korea Institute of Information and Communication Engineering, vol. 16, no. 9, (2012), pp.1970-1976.

[6] S.W. Ahn, "Smart Attendance Checking System based on BLE using a Beacon", The Journal of the Korea institute of electronic communication sciences, vol. 11, no. 2, (2016), pp.209-214.

[7] B. A. Kumar, K. C. Bhagyalakshmi, K. Lavanya and K. H. Gowranga, A Bluetooth Low Energy Based Beacon System for Smart Short Range Surveillance. 2016 IEEE International Conference on Recent Trends in Electronics, Information \& Communication Technology (RTEICT), (2016) May 20-21; Bengaluru, India.

[8] T. Zachariah, N. Klugman, B. Campbell, J. Adkins, N. Jackson and P. Dutta, The internet of things has a gateway problem. In Proceedings of the 16th International Workshop on Mobile Computing Systems and Applications (HotMobile 2015), (2015) February 12-13; Santa Fe, USA.

[9] Bluetooth Mesh Profile Specification 1.0. Bluetooth SIG, (2017).

[10] Bluetooth Mesh Model Specification 1.0. Bluetooth SIG, (2017).

[11] Y. Kwon, J. Choi and T. Song, Method and apparatus for transmitting and receiving a data in a mesh network using bluetooth. U.S. Patent Application No. 15/424,376, (2017) February 3.

[12] Uniqon. https://uniqon.com/.

[13] Bluetooth - Protocol. http://www.sharetechnote.com/html/Bluetooth_Protocol.html.

[14] Specification of the Bluetooth System - Covered Core Package version: 4.1. Bluetooth SIG, (2013)

[15] Y. Shin and S. Seol, Smartphone as a Remote Control Proxy in Automotive Navigation System, Contemporary Engineering Sciences, vol.7, no.15, (2014), pp.683-689.

[16] K. Lee, Y. Shin, J. Jo, J. Jeong, S. Choo, Y. Lee and S. Seol, Web-based Remote Control System for IPTV Service, International Journal of Control and Automation, vol.10, no.1, (2017), pp.113-122.

[17] J. Hwang, J. Jeong, Y. Shin and S. Seol, Smart home system for usability of disabled people, Convergence Research Letters, vol.3, no.4, (2017), pp.1391-1394.

[18] iBeacon - Apple developer. https://developer.apple.com/ibeacon/.

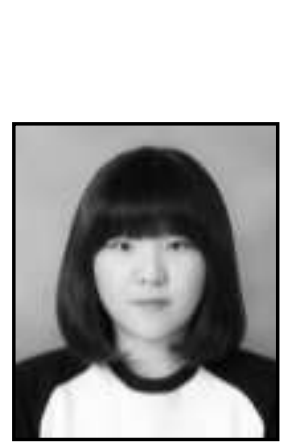

\section{Authors}

Yejin Shin, she received the B.S. and M.S degree from Korea University of Technology and Education (KOREATECH) in 2014 and 2016. She is pursuing the Ph.D. degree in Information and Communication Engineering from KOREATECH. She is interested in wireless telecommunication system, Internet of Things, RFID/USN, etc.

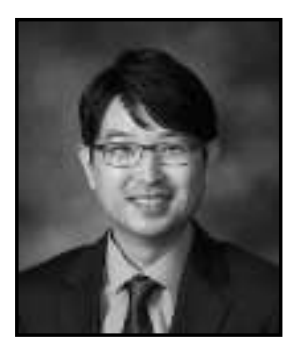

Soonuk Seol, he received his B.S. degree from Korea University of Technology and Education (KOREATECH) in 1998, M.S and Ph.D. degrees in Information and Communication Engineering from KAIST in 2000 and 2004, respectively. He worked as a senior researcher at KT from 2004 to 2012. He is currently an assistant professor in School of Electrical, Electronics, and Communication Engineering at KOREATECH. His research interests include mobile Internet, IoT, QoS, and software testing. 
International Journal of Control and Automation

Vol. 10, No. 12 (2017) 